\title{
Analysis of emotion disorders based on EEG signals of Human Brain
}

\author{
Ashish Panat ${ }^{1}$ and Anita Patil ${ }^{2}$ \\ ${ }^{1}$ Priyadarshini Indira College of Engineering \\ asishpanategmail.com \\ ${ }^{2}$ Department of Electronics \& Telecommunication, Cummins College of Engineering for \\ Women, Pune. \\ anita.patil\&@cumminscollege.in
}

\begin{abstract}
In this research, the emotions and the patterns of EEG signals of human brain are studied. The aim of this research is to study the analysis of the changes in the brain signals in the domain of different emotions. The observations can be analysed for its utility in the diagnosis of psychosomatic disorders like anxiety and depression in economical way with higher precision.
\end{abstract}

\section{KEYWORDS}

EEG, EDF format, Feature extraction, Image classifiers, Emotions, Psychosomatic disorders, Normal and excited brain, Anxiety and Depression

\section{INTRODUCTION}

A Human Brain is the organ that gives the person the capacity for art, language, rational thoughts and moral judgments. It is also responsible for each individual's personality, movements, memories, and his perception about the world. It is one of the body's biggest organs, consisting of some 100 billion nerve cells that not only put together and highly coordinated physical actions but regulate our unconscious body processes, such as digestion and breathing.

Emotions play a significant and powerful role in everyday life of human beings. Impulsive emotions express an indication of psychosomatic disorders. These disorders are reflected as the changes in the electrical activities and chemical activities in the brain. The changes can be observed by capturing the brain signals and images.

Psychiatrists nowadays, have to deal with the patients with either of two prominent psychological disorders, viz., Anxiety and Depression. Moreover, the patients are not ready to accept that the symptoms they are suffering from are indicative of some psychological disorder. It becomes a difficult job for the Psychiatrist, relatives of the patients and people around him to convince that he needs to be treated.

The proposed research is expected to quantify the psychological health of the patient from his EEG, as far as the two problems mentioned above, i.e. Anxiety and Depression are considered. 
Anxiety disorder: The term anxiety disorder covers several different forms of abnormal and pathological fear and anxiety. It covers four aspects of experiences an individual may have: mental apprehension, physical tension, physical symptoms and dissociative anxiety [1]. Anxiety disorder is divided into three types viz., anxiety disorder, phobic disorder, and panic disorder; each has its own characteristics and symptoms. They also require different treatment. The emotions present in anxiety disorders range from simple nervousness to bouts of terror. The amygdala is central to the processing of fear and anxiety, and its function may be disrupted in anxiety disorders.

Anxiety disorder is a pattern of constant worry and anxiety over many different activities and events. Other symptoms include difficulty in concentrating, fatigue, irritability, restlessness, and often becoming startled very easily [2]. A phobic disorder is a persistent fear of an object or situation. Panic disorder is an anxiety disorder characterized by recurring severe panic attacks. It may also include significant behavioral changes lasting long and ongoing worry about the implications or concern[3].

Depression: Depression is a state of low mood and aversion to activity that can affect a person's thoughts, behaviour, feelings and physical well-being [4]. Depressed people may feel sad, anxious, empty, worthless, guilty, irritable, or restless. They may lose interest in activities that once were pleasurable, experience loss of appetite or overeating, or problems concentrating, remembering details or making decisions. They may even contemplate or attempt suicide. Insomnia, excessive sleeping, fatigue, loss of energy, or aches, pains or digestive problems that are resistant to treatment may be present. The signals of the brain in these situations can also be utilized to study the emotions which can lead to great help in diagnosis of psychosomatic disorders.

The research is conducted previously to analyse the emotions by looking at the physiological aspects like users' heart rate, skin conductance and pupil dilation.

\section{LITERATURE SURVEY}

In [5] \& [6], Researchers Z. Khalili et al. have worked on Emotion detection using EEG and peripherals signals as Galvanic skin resistance, Respiration, Blood pressure, and Temperature. From these inputs, common set of features such as Mean, standard deviation and minimum and maximum of the set of data are extracted. Their research is further extended to study the improvement in the results of EEG by using correlation dimension.

In [7], the same researchers have explored on different modalities for emotion detection, such as, Visual (facial expression), Auditory (pitch, loudness, etc.), tactile (heart rate, skin conductivity etc.) and Brain signals(EEG).

In [8], Researchers have studied Brain activation during judgments of Positive emotions: Pride and Joy. They have used fMRI images for this purpose. However, recording fMRI of a brain is comparatively costly affair as compared to recording of EEG.

Researchers Arman Savran et al. [9] have also developed a technique for multimodal emotion detection. They have used the modalities like fNIRS, face video and EEG signal.

\section{BLOCK DiAgRAM}

Figure(1) shows the block diagram of the system to acquire the signals from the brain, preprocessing of the captured signals ( e.g. EEG in this study), extract the features after processing the signal, classify the processed signal and analyse it for detection of emotion disorder. 


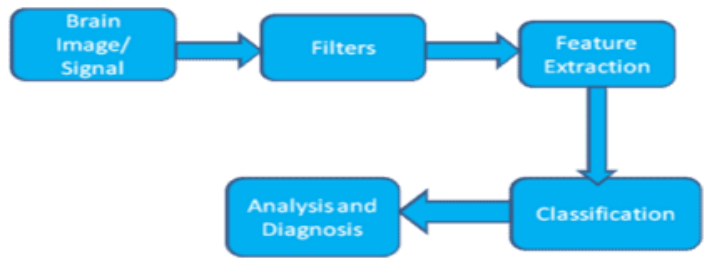

Fig.(1) Block Diagram of the system for analysis of Brain signals.

Initially, the EEG of the Brain is captured by the standard method recognized worldwide as International 10-20 system. In the Digital EEG system, these signals are first amplified and then digitized. The rate of digitization may vary from $100 \mathrm{~Hz}$ to $20 \mathrm{kHz}$, depending on the capacity of the system. Most commonly, the EEG signal captured from the EEG machine is available in the EDF format (European Data Format). It must be first converted into .Wav format which is suitable for processing. The signals are then filtered. The pass band of the filter depends on the frequency of the interest for that particular signal, e.g. a low pass filter for Delta and Theta waves, a band pass filter for alpha waves, and a high pass filter for gamma waves etc.

After filtering the signal, the features can be extracted, which can be compared and used for further analysis.

\section{Capturing The Signal}

The fig.(2) shows the placement of the electrodes for capturing the EEG.

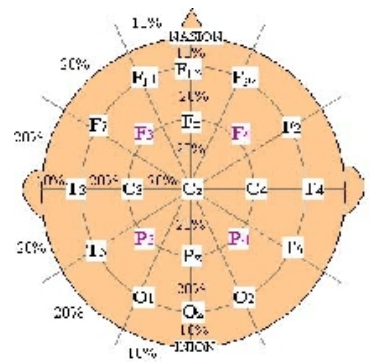

Fig (2) Placement of electrodes for EEG [12]

As per the International Standard, known as international 10-20 system, 19 electrodes are connected to different locations on the scalp, which are salient points from the clinical point of view, and one reference electrode is connected to ground. Whenever, the detailed study of EEG is intended in case of some patients, or for the research purpose, the number of electrodes may increase up to 256 also. In case of infants, i.e. neonatal EEG, the number can be decreased.

\section{Preprocessing}

The EEG signal from the EEG machine is available in the EDF format (European Data Format). It is first converted into.Wav format which is suitable for processing. The software EDF2WAV is used for this purpose. These WAV files can be directly referred into MATLAB programs, as input data. The different frequency bands. The Fig. (3) shows the flowchart of different steps of Pre-processing followed by feature extraction. 
International Journal of Computer Science, Engineering and Applications (IJCSEA) Vol.2, No.4, August 2012

$\begin{aligned} & \text { EDF format } \\ & \text { EEG signal }\end{aligned} \rightarrow \begin{aligned} & \text { EDF to WAV } \\ & \text { Convertor }\end{aligned} \rightarrow \begin{aligned} & \begin{array}{l}\text { Read WAV files } \\ \text { in MATLAB }\end{array} \\ & \text { Signals }\end{aligned}, \begin{aligned} & \text { Separate differen } \\ & \text { signals }\end{aligned} \rightarrow \begin{aligned} & \text { Extrast } \\ & \text { fectures }\end{aligned}$

Fig.(3) Pre-processing and feature extraction

\section{Feature Extraction}

After pre- processing, the following features are extracted from this signal: Mean, Standard deviation, skewness, kurtosis, mean of absolute values of first difference of raw signals, mean of absolute values of first difference of normalized signal [5]. The images of these signals can be stored for further analysis. For feature extraction, basic mathematical formulae for mean, variance etc. can be applied. Also, one can use the wavelet transform for this purpose.

Comparison of these features can be made to find the emotion disorder. As a first step towards detection of emotional disorder, one obvious symptom, seizure, is identified by comparing EEGs of a patient in normal condition \& with seizure.

Seizure is one of the symptoms that may occur in a patient suffering from anxiety. The abnormal activity of the brain in epileptic patient is known as seizure condition. This activity appears on the screen of the EEG machine as waveforms of varying frequency and amplitude measured in voltage. In this study, two features, viz., Mean and Variance are compared to detect the seizure occurred in the patient.

\section{RESULTS}

The following snap shots from MATLAB result windows show the conversion of EDF file (Fig.4) to .WAV file (Fig.5), band pass filter applied to theta wave Fig.(6).

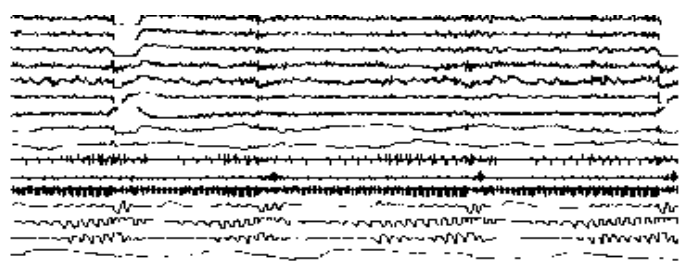

Fig.(4) Signal extracted from .EDF file.

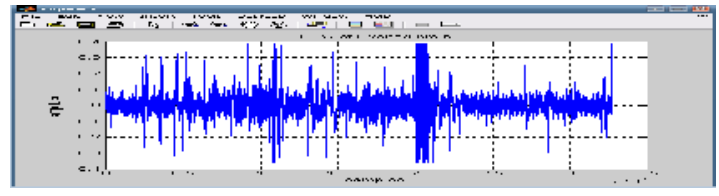

Fig.(5) Result of MATLAB code for EEG in .WAV format

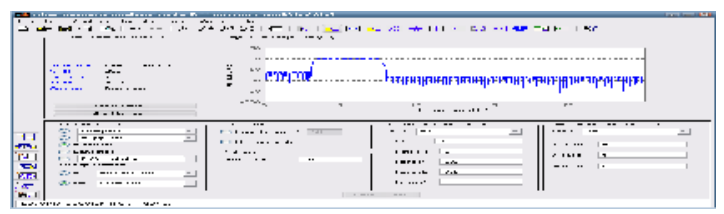

Fig(6) Result of Band pass filter for Theta wave (using FDA tool) 
International Journal of Computer Science, Engineering and Applications (IJCSEA) Vol.2, No.4, August 2012

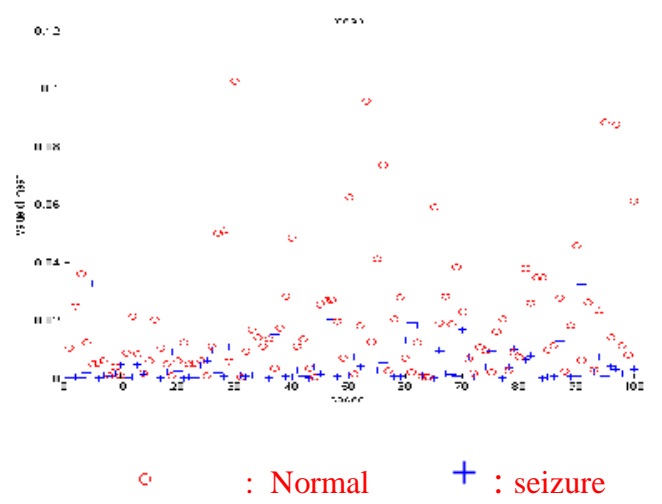

Fig.(7) Plot of Mean for Normal and seizure condition

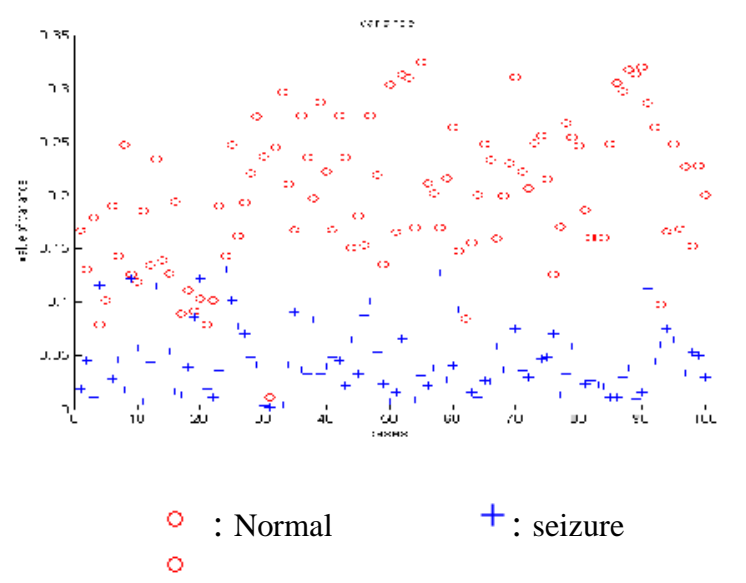

Fig.(8) Plot of Variance from EEG signal recorded for Normal and seizure condition

Result Table

\begin{tabular}{|c|c|}
\hline Feature & Accuracy \\
\hline Mean & $75 \%$ \\
\hline Variance & $93 \%$ \\
\hline
\end{tabular}

Table 1: Comparison of results of two features, Mean and Variance

\section{CONCLuSion}

The study has proved the effective utility of economical and simple method of study of brain using EEG for diagnosis the different emotion disorders viz., anxiety and depression. One of the major symptoms, seizure is analysed in this study. The EEG signal in EDF format is converted into .WAV format using EDF to WAV converter. The signal is then passed through the filters of different frequencies to separate alpha, beta, delta and theta waves. The features are extracted from these signals. After analyzing Mean, Power Spectral Entropy and Variance of both normal and seizer EEG signals, it was found that using Variance gives more accurate information about the seizure in EEG signal among the given feature sets.

This technique has revealed the possibility of precise diagnosis of psychosomatic disorders in more simple and economical way. 


\section{REFERENCES}

[1] David Healy, Psychiatric Drugs Explained, Section 5: Management of Anxiety, Elsevier Health Sciences, 2008, pp. 136-137

[2] www.ncbi.nlm.nih.gov/pubmedhealth

[3] www.wikipedia.org/wiki/Phobi

[4] Kessler RC, Chiu WT, Demler O, Merikangas KR, Walters EE (June 2005). "Prevalence, severity, and comorbidity of 12-month DSM-IV disorders in the National Comorbidity Survey Replication". Arch. Gen. Psychiatry 62 (6): 617-627. doi:10.1001/archpsyc.62.6.617. PMC 2847357. PMID 15939839.

[5] Emotion detection using brain and peripheral signals: Z. Khalili1, M. H. Moradi Biomedical Engineering Faculty, Amirkabir University of Technology, Tehran, Iran. Proceedings of the CIBEC'08 978-1-4244-2695-9/08/\$25.00 @2008 IEEE.

[6] EEG Model and Location in Brain when at Emotion Recognition System Using Brain and Peripheral Signals using Correlation Dimension to Improve the Results of EEG: Z. Khalili , M. H. Moradi Biomedical Engineering Faculty, Amirkabir University of Technology, Tehran, Iran. 978-1-42443553-1/09/\$25.00 @2009 IEEE.

[7] Special Issue on Multimodal affective Interaction : IEEE Transaction on Multimedia, vol.12.,no.6., October 2010.

[8] Brain Activation during Judgments of Positive Self-conscious emotion and Positive Basic Emotion: Pride and Joy : by Hidehiko Takahashi, Masato Matsuura et al in Cerebral Cortex April 2008; 18; 898-903.

[9] Emotion Detection in Loop from Brain signals and Facial Images by Arman Savran, Koray Ciftci,etc.: Enterface'06, July17th, Dubrovnik, Croatia.

[10] EMcLachlan (2004) Discriminant Analysis and Statistical Pattern Recognition In: Wiley Interscience

[11] Bremner D, Demaine E, Erickson J, Iacono J, Langerman S, Morin P, Toussaint G (2005). "Outputsensitive algorithms for computing nearest-neighbour decision boundaries". Discrete and Computational Geometry 33 (4): 593-604.

Conference in which this paper was published- DPPR2012-Advance Computing and Information Technology, held on13-14 July 2012, at Chennai, India. Paper id : 12.

\section{Authors: ****}

1. Dr. Ashish Panat has completed her B.E (Electronics) in the year 1987, his M.E (Electronics) in 1998 and Phd in "Study of Intelligent thinking machines" in 2009. He has has worked as a Lecturer in SSGMCOE, Shegaon, PRMITR, Badnera, DIMT, Raipur, College of Administrative Services, Lutor University UK,Oman. Currently he is working as a Principal in Priyadarshani Indira College of Engineering, Nagpur. He has a total experience of 23 years. He is associated in IEEE Conferences and IJMIA

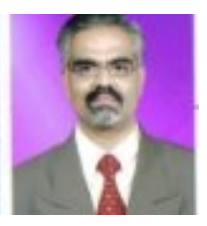
conference. He is editor in Chief of Series Publications with ISSN No.0976-6227. He has received grants from RPF-AICTE. He has published 2 books titled "Fundamentals of Physics". He has presented more than 15 papers in National and International Conferences. She is an IEEE member and a Life member of IETE, CSI and ISTE. His area of interest is in Speech Processing, Signal Processing and Intelligent systems.

2. Anita Patil, is presently working as Asssistant Professor at Cummins College of Engineering for Women, Pune. She has completed her Master's degree in Electronics from Mumbai University, in 1999. She has a teaching experience of 21 years. She is pursuing her Ph.D. in the field of Biomedical Image Processing under the Guidance of Dr. Ashish Panat. She has presented total 5 papers in various International conferences and 2 papers in National conferences.

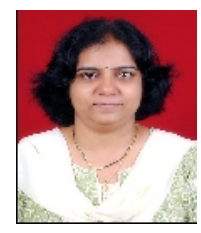

